\title{
Properties of simulated sunspot umbral dots
}

\author{
L. Bharti, B. Beeck, and M. Schüssler
}

\author{
Max-Planck-Institut für Sonnensystemforschung, Max-Planck-Str. 2, 37191 Katlenburg-Lindau, Germany \\ e-mail: bharti@mps.mpg.de
}

Received 21 September 2009 / Accepted 18 November 2009

ABSTRACT

\begin{abstract}
Realistic 3D radiative MHD simulations reveal the magneto-convective processes underlying the formation of the photospheric fine structure of sunspots, including penumbral filaments and umbral dots. Here we provide results from a statistical analysis of simulated umbral dots and compare them with reports from high-resolution observations. A multi-level segmentation and tracking algorithm has been used to isolate the bright structures in synthetic bolometric and continuum brightness images. Areas, brightness, and lifetimes of the resulting set of umbral dots are found to be correlated: larger umbral dots tend to be brighter and live longer. The magnetic field strength and velocity structure of umbral dots on surfaces of constant optical depth in the continuum at $630 \mathrm{~nm}$ indicate that the strong field reduction and high velocities in the upper parts of the upflow plumes underlying umbral dots are largely hidden from spectro-polarimetric observations. The properties of the simulated umbral dots are generally consistent with the results of recent highresolution observations. However, the observed population of small, short-lived umbral dots is not reproduced by the simulations, possibly owing to insufficient spatial resolution.
\end{abstract}

Key words. magnetic fields - Sun: photosphere - sunspots

\section{Introduction}

Recent realistic MHD simulations have revealed that the complex brightness and flow structure of sunspots results from convective energy transport dominated by a strong vertical (in the umbra) or inclined (in the penumbra) magnetic field (Heinemann et al. 2007; Scharmer et al. 2008; Rempel et al. 2009b,a). In the umbra, such magneto-convection occurs in the form of narrow upflow plumes with adjacent downflows (Schüssler \& Vögler 2006, henceforth referred to as SV06). In computed intensity images, these plumes appear as bright features that share various properties with observed umbral dots. Here we provide a systematic study of the umbral simulation results with specific emphasis on the properties of the bright structures and their comparison with recent results from high-resolution observations.

\section{MHD simulation}

We used the MURaM code (Vögler 2003; Vögler et al. 2005) with nearly the same simulation setup as SV06. The dimensions of the computational box are $5.76 \mathrm{Mm} \times 5.76 \mathrm{Mm}$ in the horizontal directions and $1.6 \mathrm{Mm}$ in the vertical. Rosseland optical depth unity is about $0.4 \mathrm{Mm}$ below the upper boundary. The grid cells have a size of $20 \mathrm{~km}$ in the horizontal directions and $10 \mathrm{~km}$ in the vertical. The magnetic diffusivity is $2.8 \times 10^{6} \mathrm{~m}^{2} \mathrm{~s}^{-1}$, the lowest value compatible with the grid resolution. The hyperdiffusivities for the other diffusive processes (for details, see Vögler et al. 2005) in the deepest layers of the box were chosen lower than the values used by SV06 in order to minimize their contribution to the energy transport. Since we do not synthesize spectral lines or Stokes profiles, the radiative transfer can be treated in the gray approximation. The fixed vertical magnetic flux through the computational box corresponds to a mean field strength of $2500 \mathrm{G}$. Side boundaries are periodic, and the top boundary is closed for the flow. The lower boundary is open and the thermal energy density of the inflowing matter has been fixed to a value of $3.5 \times 10^{12} \mathrm{erg} \mathrm{cm}^{-3}$, leading to a total surface energy output comparable to that of a typical sunspot umbra, i.e., about $20 \%$ of the value for undisturbed solar surface. The magnetic field is assumed to be vertical at the top and bottom boundaries.

We started our simulation from the last snapshot of the run analyzed by SV06 and continued for $10.8 \mathrm{~h}$ solar time in order to obtain a sufficiently large statistical sample of simulated umbral dots. Since the average magnetic field in the simulation is vertical, our results represent the inner part of a sunspot umbra with central umbral dots - as opposed to peripheral umbral dots, which often are related to penumbral filaments (e.g., Grossmann-Doerth et al. 1986).

\section{Image segmentation}

To obtain statistical properties of the large number of simulated umbral dots (UDs), we used an automated detection method to distinguish between bright features and the surrounding dark background (DB). Since both the UDs and the background cover a broad range of intensities, we chose the MLT (Multi Level Tracking) algorithm of Bovelet \& Wiehr (2001) as the basis of our image segmentation. This method has been successfully applied to various observational data (e.g., Wiehr et al. 2004; Bovelet \& Wiehr 2007, 2008; Kobel et al. 2009), including highresolution images of sunspot umbrae (Riethmüller et al. 2008b).

The basic concept of the MLT algorithm is to segment the image by applying a sequence of intensity thresholds with decreasing values, keeping all features already detected. In our case, a complication arises from the dark lanes shown by many of our simulated UDs. This can lead the algorithm to split an UD into two parts (see Fig. 1). In order to avoid such undesired splitting, we permit the merging of segments that were separate for threshold $n$ after applying threshold level $n+1$ provided that 


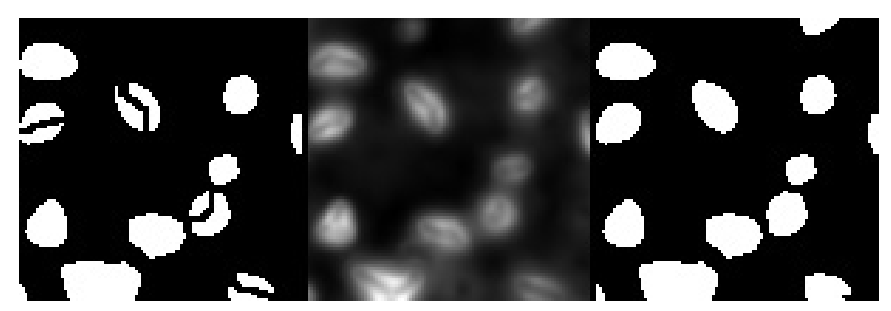

Fig. 1. Over-segmentation of simulated umbral dots due to dark lanes. The section of the original image (middle panel) shows umbral dots with dark lanes. The unmodified MLT algorithm leads to an undesired splitting of many dots in the segmented image (left panel). Introducing a modification of the algorithm controlling the merging of segments removes the problem in most cases (right panel).

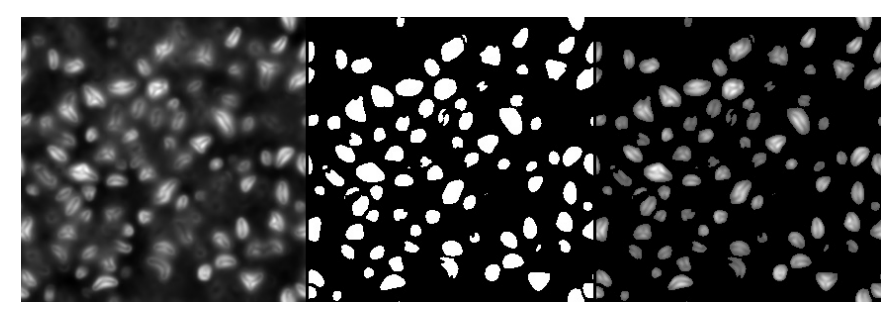

Fig. 2. Result of the MLT image segmentation. Left: original image; middle: segmented image (binary map); right: segmented umbral dot areas with their original intensities.

their maximum intensity does not exceed a value equal to the latter threshold multiplied by a suitably chosen factor $c$. For our analysis, we used 15 intensity thresholds ranging from about 2.5 to 1.5 times the intensity of the dark background (somewhat depending on the dataset used, see Sect. 4.2) and a value of $c=1.45$, which suppresses most cases of unwanted splitting of UDs.

In a final step of the segmentation process, we removed from the list of UDs all segmented structures whose maximum intensities do not exceed the lowest threshold by at least $10 \%$; such features are considered to be mere fluctuations of the background. Figure 2 shows an example of the resulting segmentation of a typical simulated image.

In order to study UD evolution and lifetimes, we extended the MLT algorithm to include time, such that the segmentation can be carried out in three dimensions (two spatial and one temporal) to follow the temporal evolution of individual UDs.

\section{Results}

\subsection{Overall temporal evolution}

The relative inefficiency of the convective energy transport in the strong umbral magnetic field leads to a rather long thermal relaxation time of the system, so that it can take hours until it settles down into a statistically stationary state. With our long simulation run of $10.8 \mathrm{~h}$ solar time, we were able to follow the overall changes of the system throughout the relaxation phase.

The steady decrease of the total internal energy in the computational box shown in Fig. 3 (left panel) clearly indicates that the sytem is not relaxed until about 6-7 h after the start of the simulation. Comparing the time-integrated total radiative output from the box with the integrated decrease of the total internal energy given in the right panel of Fig. 3 suggests that during the first half of the evolution the energy output was mostly covered by a loss of internal energy, i.e., the plasma in the box cooled.
Only after about $6 \mathrm{~h}$, the two curves start to diverge and the internal energy does no longer decrease. At about the same time, the slope of the integrated energy output steepens, indicating a somewhat higher energy output. This is confirmed by Fig. 4 (left panel), which shows that the total radiative flux is higher by about $10 \%$ in the second half of the run.

These results suggest that the character of the energy supply has changed halfway through the run: while the radiated energy was taken from the internal energy in the first half and was not significantly replenished through convective inflows through the bottom of the box, convection became more effective in the second half of the run and led to a thermally relaxed stationary situation with a somewhat higher total radiative output. This interpretation is supported by considering the time evolution of kinetic energy (Fig. 4, right panel), which shows an increase by a factor of 3 in the second half of the run. The magnetic energy also grows slightly, by about $10 \%$, probably due to the stronger fluctuations (local compressions of the magnetic flux) caused by the higher velocities.

\subsection{Statistical properties of umbral dots}

The existence of the two phases (cooling phase and convective phase) in the temporal evolution of the umbra simulation is also reflected in differences of the average UD properties in both phases. The snapshots of (bolometric) brightness in Fig. 5 indicate that larger UDs appear in the second phase while the total number of UDs decreases. We therefore defined separate datasets for each phase and analyzed them individually. The first part $(\mathrm{P} 1)$ covers the time between $0.5 \mathrm{~h}$ and $4.5 \mathrm{~h}$ after the beginning of the run while the second part (P2) extends from $6.2 \mathrm{~h}$ to $10.3 \mathrm{~h}$. We excluded the transition between the two phases and only kept periods for which the average UD properties do not show significant secular trends.

For the MLT segmentation considered in this section, we used maps of the bolometric (frequency-integrated) emergent intensity in the vertical direction. The maps are separated in time by about 1 min each, so that UDs can be analyzed in a statistical manner as well as their evolution followed in time. Owing to the different characteristics of the UDs, slightly different MLT threshold levels were chosen for P1 and P2, respectively. For dataset P1, the 15 intensity thresholds range between $20 \%$ and $31 \%$ of the average quiet-Sun brightness while for P2 they range from $22 \%$ to $35 \%$.

Figure 6 shows, separately for P1 and P2, the temporal evolution of various average quantities of the segmented UDs and of the remaining domain area, the DB. The area fraction of UDs (top left panel) tends to be somewhat higher in P2 compared to P1; its relatively strong fluctuations result from the relatively small size of the computational box. In P2, there are less (top right panel) but, on average, larger (middle right panel) and brighter (middle left and bottom left panel) UDs, as already indicated by the snapshots shown in Fig. 5. The DB is also slightly brighter in P2 (bottom left panel). The mean bolometric intensity of the UDs approaches roughly stationary values of about $0.23(\mathrm{P} 1)$ and $0.26(\mathrm{P} 2)$ of the quiet-Sun value, or about $1.6(\mathrm{P} 1)$ and $1.7(\mathrm{P} 2)$, respectively, in units of the corresponding DB intensity (bottom right panel). These results indicate that the upflows underlying the UDs contribute more strongly to the convective energy transport in $\mathrm{P} 2$. This includes not only the fraction of the radiative output directly covered by the UDs (about $30 \%$ ), but also the horizontal radiative losses of the upflow plumes, which heat their environment and thus contribute to the energy output of the DB. The higher average brightness of the DB in the 
L. Bharti et al.: Properties of simulated sunspot umbral dots
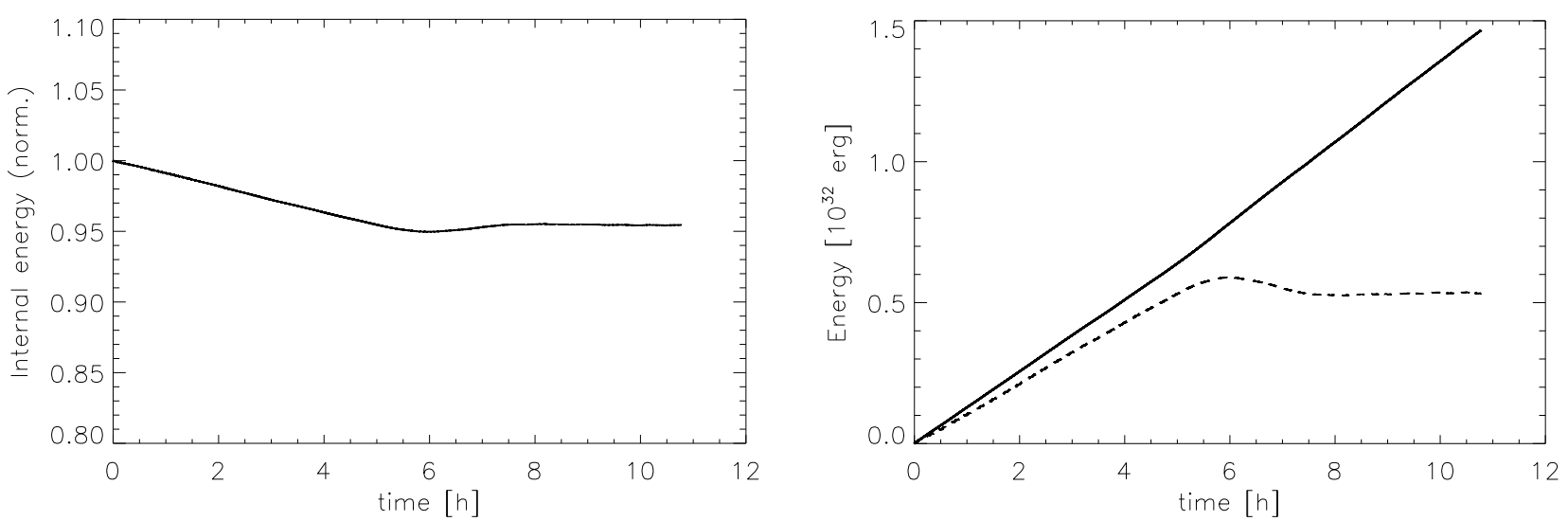

Fig. 3. Thermal relaxation of the system. Left panel: time evolution of the total internal energy (normalized to its initial value); right panel: total (radiative) energy output (solid line) and loss of total internal energy (dashed line), both integrated in time from $t=0$ onward.
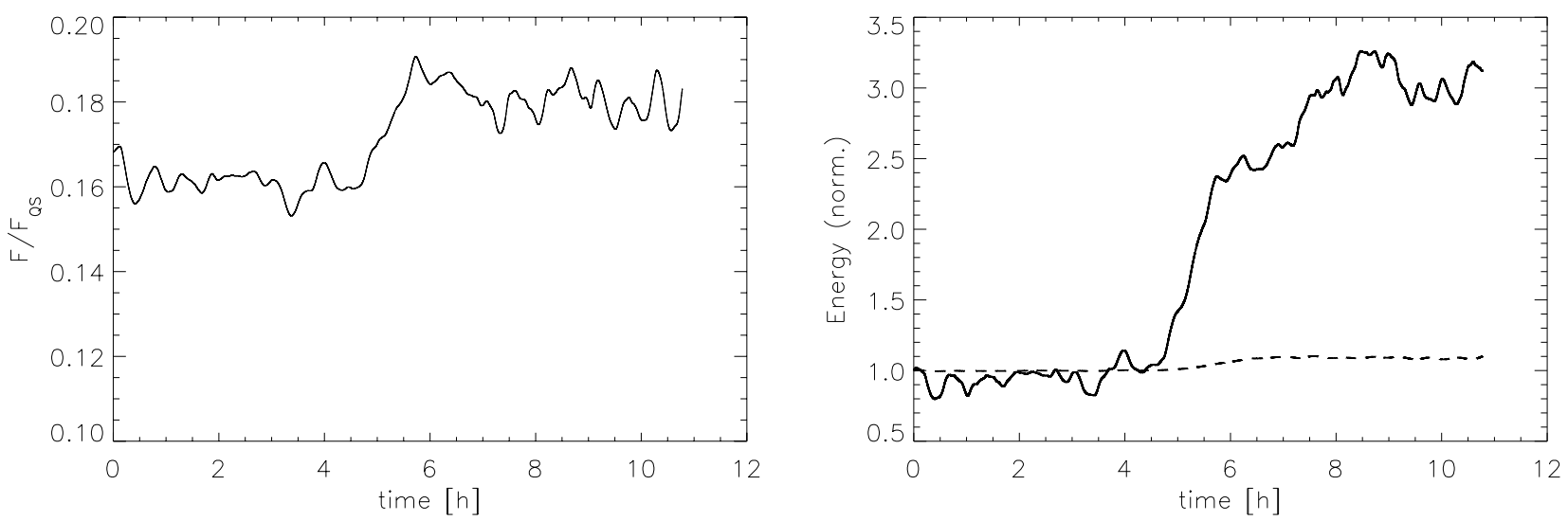

Fig. 4. Temporal evolution of the system. Left panel: radiative energy flux from the box, normalized by the corresponding value for the same area of undisturbed solar photosphere; right panel: total kinetic energy (solid line) and magnetic energy (dashed line), both normalized to their values at $t=0$.
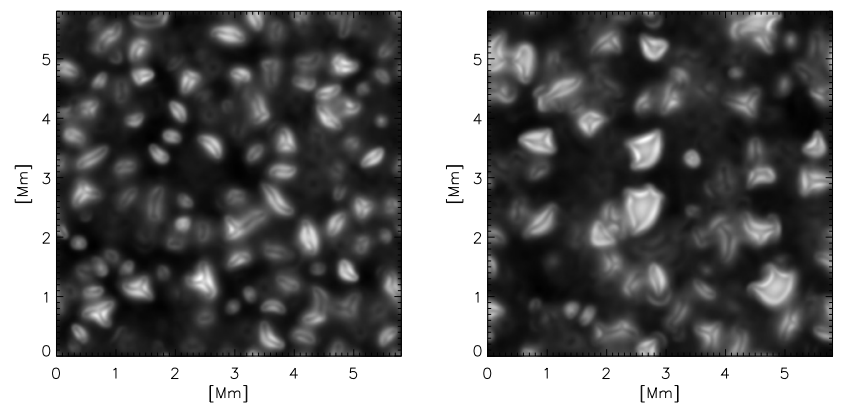

Fig. 5. Snapshots of the bolometric emergent intensity from the two phases of the simulation. Upper panel: initial cooling phase $(t \simeq 2 \mathrm{~h})$; Lower panel: convective phase $(t \simeq 9 \mathrm{~h})$.

second phase (P2) is thus consistent with a bigger contribution of the UDs to the overall convective energy transport.

The average UD area resulting from our simulation and segmentation analysis is $0.08 \mathrm{Mm}^{2}$ for $\mathrm{P} 1$ and $0.14 \mathrm{Mm}^{2}$ for $\mathrm{P} 2$, corresponding to average diameters of $320 \mathrm{~km}$ and $420 \mathrm{~km}$ under the assumption of circular areas. These values are higher than those reported in recent observational studies of UDs with the 1-m SST on La Palma: Riethmüller et al. (2008b) give an average area of $0.04 \mathrm{Mm}^{2}$, Sobotka \& Hanslmeier (2005) estimate $0.025 \mathrm{Mm}^{2}$ while Sobotka \& Puschmann (2009) even find a value near $0.01 \mathrm{Mm}^{2}$. On the other hand, the UDs with dark lanes studied by the latter authors have an average area around $0.08 \mathrm{Mm}^{2}$ and even larger UDs have been studied by Bharti et al. (2007b). Also, Watanabe et al. (2009a) find an average area of about $0.1 \mathrm{Mm}^{2}$ from Hinode data. In such a comparison one has to take into account that the lowest intensity thresholds in our MLT segmentation are not far above the DB values, while the observational analyses typically define the edges of an UD by the half-width of the local intensity contrast or by the inflection point of the intensity profile. These procedures tend to yield smaller areas than our approach, which covers the full extension of the UDs (cf., for example, the UD outlines shown in Figs. 5 and 6 of Riethmüller et al. 2008b). We tested this conjecture by applying the procedure of Riethmüller et al. (2008b) to a few representative snapshots in our datasets. We found that the resulting UD areas are, on average, about 50\% smaller than the values determined by our MLT procedure. This brings the simulation and the observational results into rough agreement - the simulations possibly lacking a population of very small, shortlived UDs (Sobotka \& Puschmann 2009).

For the full datasets P1 and P2, Fig. 7 shows area histograms of the bolometric intensity and the vertical component of the magnetic field at constant height $z=0$, which corresponds to the mean level of Rosseland optical depth unity. Histograms for UDs and DB are given separately (including all image pixels belonging to each class). The UD intensity distributions reflect the 

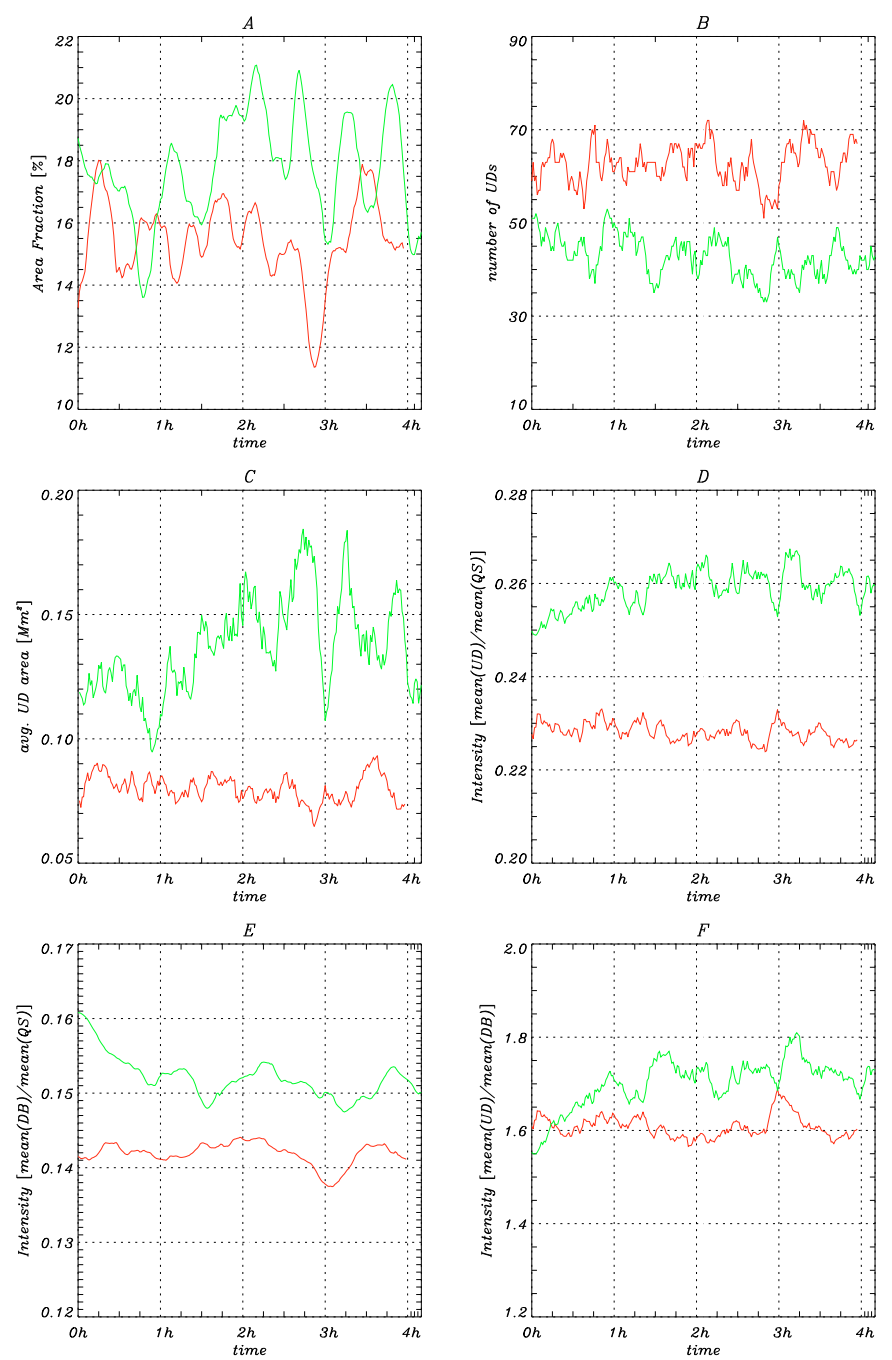

Fig. 6. Time evolution of UD properties, given separately for the two phases P1 (cooling phase, red curves) and P2 (convective phase, green curves). $A$ : UD area fraction; $B$ : number of UDs; $C$ : average UD area; $D$ : mean bolometric UD intensity (normalized by the mean quiet-Sun value); $E$ : average bolometric intensity of $\mathrm{DB}$ (normalized by the mean quiet-Sun value); $F$ : mean bolometric UD intensity (normalized by the corresponding average for the DB). Time is counted from the start of the respective dataset $(0.5 \mathrm{~h}$ and $6.2 \mathrm{~h}$ after the start of the simulation for $\mathrm{P} 1$ and $\mathrm{P} 2$, respectively).

fact that UDs tend to be brighter in P2. The tail of DB intensities overlapping with the UD distribution results from the "trenches" generated by the MLT algorithm to separate closely neighboring or clustered UDs and the exclusion of the faintest UDs.

The area histograms of the vertical magnetic field given in the lower panel of Fig. 7 shows a very broad distribution of field strength in the UDs, ranging from slightly negative values up to about $3000 \mathrm{G}$. While the field strength becomes very low owing to expansion and flux expulsion in the core of the rising plumes that generate the UDs, horizontal radiative heating extends the wings of the bright intensity structure (i.e., the UD) into the surrounding umbra with high field strength. Since we segment the images according to the intensity structure, the peripheries of the so-defined UDs harbor strong magnetic fields. Negative field strength arises when a strong downflow catches and drags a magnetic field line, so that it is stretched into a hairpin-like shape (see Fig. 14 and Sect. 4.3). The average field strength in the DB
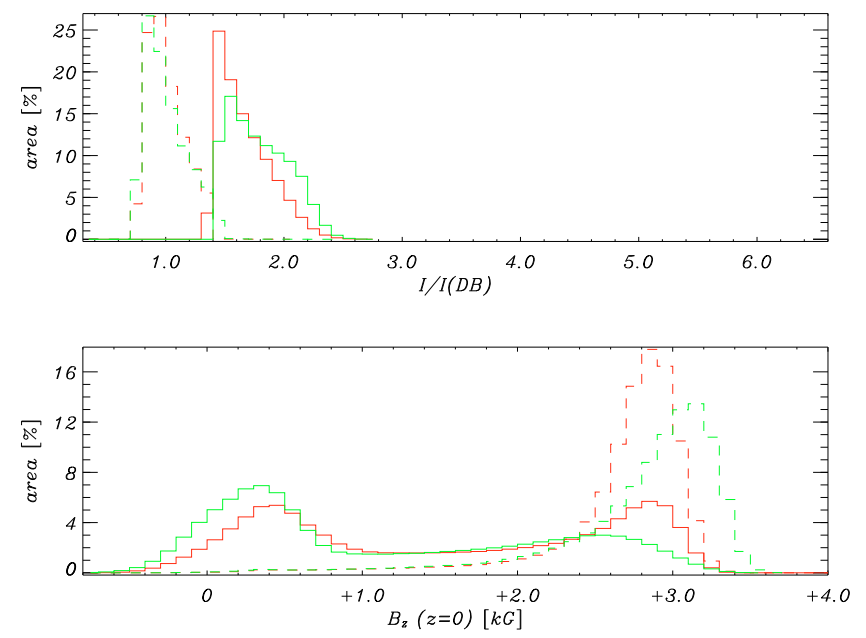

Fig. 7. Area histograms of bolometric intensity (upper panel) and vertical field strength (lower panel). The field strength is given taken at $z=0$, corresponding to the average geometrical height level of Rosseland optical depth unity. Solid curves refer to UDs, dashed curves to DB. Red and green curves indicate datasets $\mathrm{P} 1$ and $\mathrm{P} 2$, respectively.
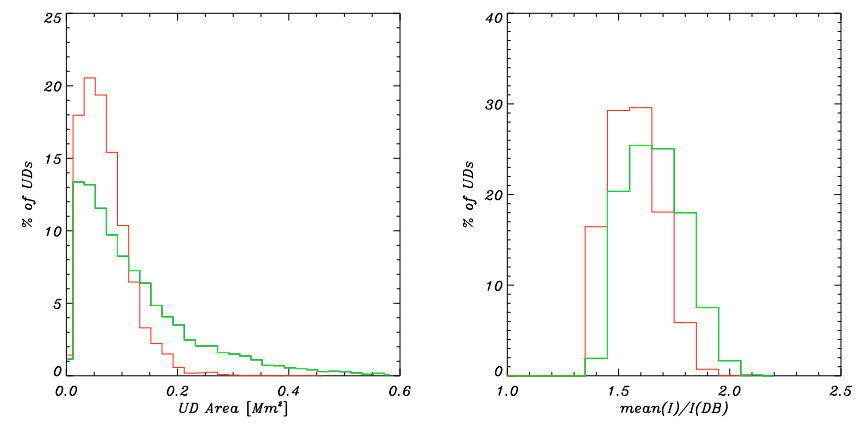

Fig. 8. Histograms of UD area (left) and mean bolometric brightness (right, normalized to the average DB) for all segmented maps. Red and green curves refer to datasets $\mathrm{P} 1$ and $\mathrm{P} 2$, respectively.

is higher in P2 since the larger area fraction of UDs compresses the magnetic flux in the DB.

The distributions of UD area and mean brightness for all segmented maps (with one minute cadence, so that UDs are considered more than once, in various stages of their evolution) are illustrated by the histograms shown in Fig. 8. UDs with areas below $0.02 \mathrm{Mm}^{2}$ were omitted as most of them represent fluctuations in the DB. The area distribution for $\mathrm{P} 2$ is broader, with less smaller and more larger UDs than for P1. The largest UDs have areas of $0.44 \mathrm{Mm}^{2}$ and $0.79 \mathrm{Mm}^{2}$ for $\mathrm{P} 1$ and $\mathrm{P} 2$, respectively. The distribution of average UD bolometric brightness contrast (right panel of Fig. 8) is shifted towards higher values for P2, the mean values being $1.62(\mathrm{P} 1)$ and $1.72(\mathrm{P} 2)$.

Since the MLT tracking algorithm identifies and tracks UDs during their whole life cycle, we determined lifetimes for 558 UDs from P1 and 500 UDs from P2. The lifetime distributions are shown in the form of histograms in Fig. 9 (excluding the roughly $10 \%$ of the UDs with lifetimes below 8 min, which seem to constitute a separate group of low-amplitude fluctuations). The lifetime distributions are similar for both datasets, with average values of $28.2 \mathrm{~min}(\mathrm{P} 1)$ and $25.1 \mathrm{~min}(\mathrm{P} 2)$. The mean lifetimes reported from observations range from 3 min to about an hour, with recent high-resolution observations typically indicating rather short mean lifetimes below 10 min (e.g., Kusoffsky \& Lundstedt 1986; Ewell 1992; Sobotka et al. 1997; 


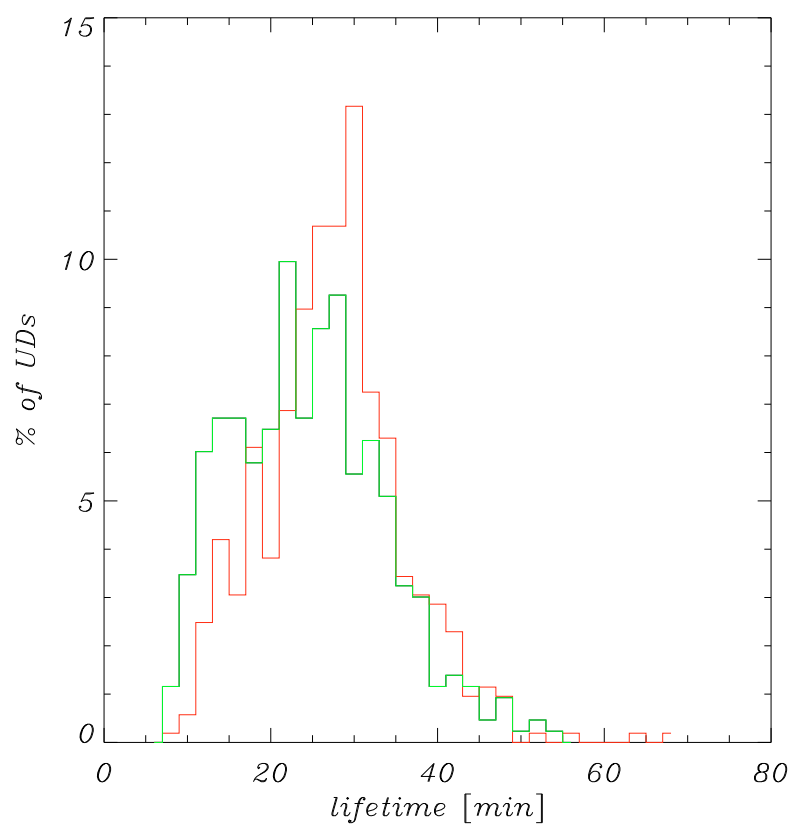

Fig. 9. Histogram of lifetimes for UDs that could be tracked during their whole evolution. Red and green curves refer to datasets P1 and P2, respectively.
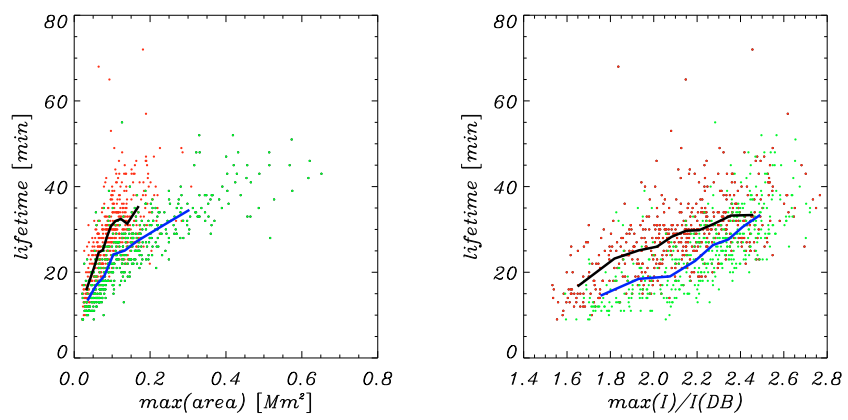

Fig. 10. Relationship between UD lifetime, area and brightness. Left: scatter plot of lifetime vs. maximum area; right: scatter plot of lifetime vs. maximum area-averaged bolometric brightness reached during the lifetime of an UD (normalized by the average brightness of the DB). Red and green dots refers to datasets P1 and P2, respectively; solid black (P1) and blue (P2) lines connect binned values for 50 points each.

Kitai et al. 2007; Riethmüller et al. 2008b; Sobotka \& Puschmann 2009; Watanabe et al. 2009a). However, similar to our simulation results, the distribution of lifetimes from any observation is rather broad and "typical" lifetimes cannot be defined easily (Socas-Navarro et al. 2004). Taken at face value, the observations indicate the existence of a large number of shortlived UDs that are not shown by the simulations. However, it is also possible that some observational lifetimes are affected by brightness fluctuations of UDs and by seeing effects.

The relationship between lifetime and maximum UD area and brightness in the course of the UD evolution is shown in Fig. 10 in the form of scatter plots with curves connecting binned values. Although there is a significant amount of scatter, the plots indicate that longer-lasting UDs tend to be larger and brighter. A qualitatively similar lifetime-size relation is reported by Riethmüller et al. (2008b) for UDs with lifetimes below 20 min while longer-lived UDs are not found to be systematically larger.
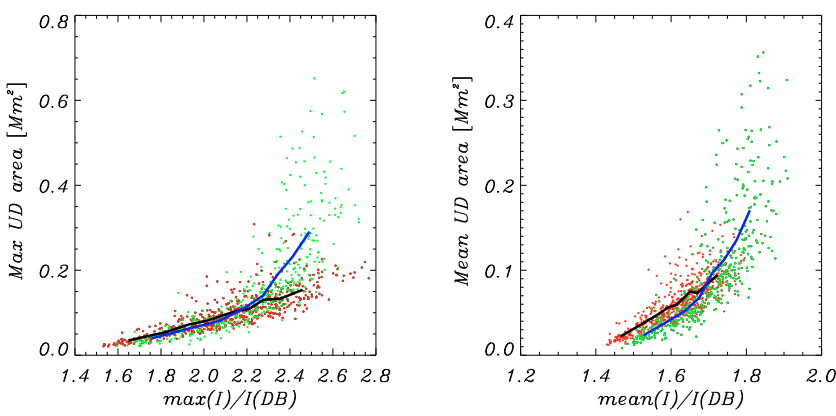

Fig. 11. Relation between area and brightness for the same set of UDs as in Fig. 10. Left: scatter plot of maximum area vs. maximum (normalized) bolometric brightness during the lifetime of an UD; Right: scatter plot of mean UD area (over UD lifetime) vs. mean area-averaged brightness (over UD lifetime). Red and green color refers to datasets P1 and $\mathrm{P} 2$, respectively; solid black (P1) and blue (P2) lines connect binned values for 50 points each.

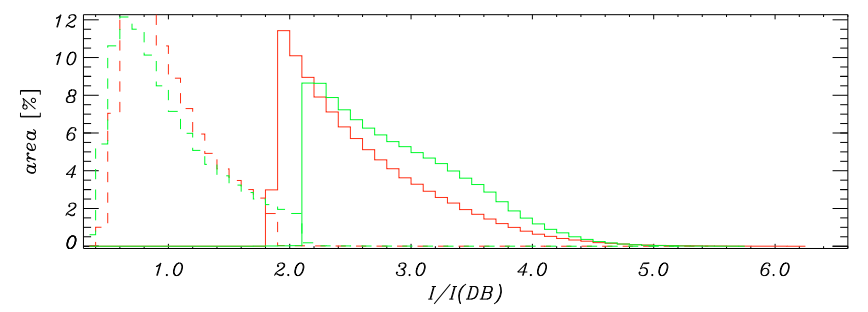

Fig. 12. Area histograms of the continuum intensity at $630 \mathrm{~nm}$. Solid curves refer to UDs, dashed curves to DB. Red and green curves indicate datasets $\mathrm{P} 1$ and $\mathrm{P} 2$, respectively.

The lifetime-area and lifetime-brightness correlations are probably due to the fact that stronger and more extended convective upflows are maintained longer and create larger and brighter UDs: higher upflow speed entails a bigger convective energy flux density (brighter UDs) and also more mass flux and kinetic energy available for the sideways expansion of the upflow plume (bigger UDs). This explanation is in line with the relationship between UD area and (maximum and mean) brightness shown in Fig. 11. Similar trends were found observationally by Tritschler \& Schmidt (2002) and by Sobotka \& Puschmann (2009) while Riethmüller et al. (2008b) do not find a clear relationship.

\subsection{UD properties and optical-depth dependence at $630 \mathrm{~nm}$}

The iron line pair near $630 \mathrm{~nm}$ and the nearby continuum is often used for observations of sunspot fine structure. In order to compare with observational results derived from such data, we considered $57(\mathrm{P} 1)$ and $74(\mathrm{P} 2)$ snapshots from our simulation and calculated images of the (vertically emerging) continuum intensity at $630 \mathrm{~nm}$. These images were segmented by the MLT procedure to obtain maps of UDs. In addition to the UD properties studied in the previous section, we correlated the UD maps with the distributions of vertical magnetic field and vertical velocity on surfaces of equal optical depth at $630 \mathrm{~nm}$. A full synthesis of line profiles and Stokes parameters for a detailed comparison with observed spectro-polarimetric data is beyond the scope of this work and will be carried out in a forthcoming paper (Vitas, Vögler \& Keller, in preparation).

Area histograms for the continuum intensity at $630 \mathrm{~nm}$ are shown in Fig. 12. Compared to the histograms of the bolometric intensity shown in Fig. 7, the intensity contrasts between UDs and DB is much higher, resulting from the fact that $630 \mathrm{~nm}$ is on 


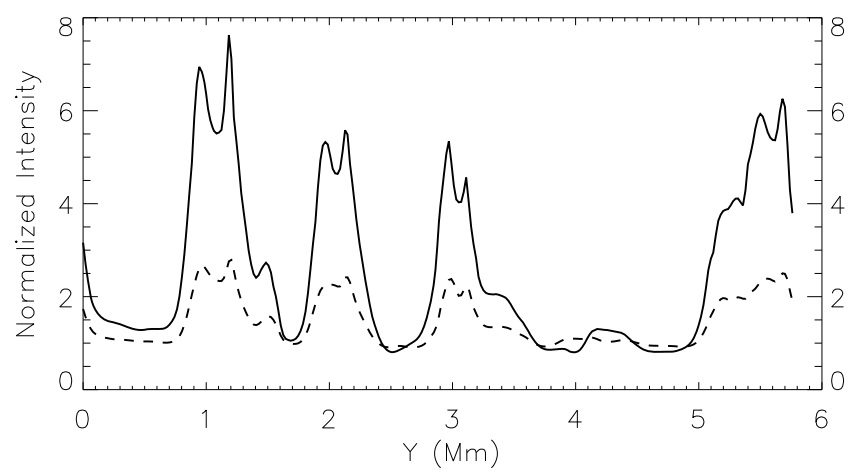

Fig. 13. Profiles of vertically emerging intensity along a cut at $x=$ 4.6 Mm through the image shown in the right panel of Fig. 5. The solid line refers to the continuum intensity at $630 \mathrm{~nm}$, the dashed line to the bolometric intensity. Intensities are normalized individually by the corresponding local DB intensities.

the blueward side from the maximum of the respective Planck functions. The significantly higher contrast between DB and UD is also illustrated by the intensity profiles shown in Fig. 13. The mean UD intensity values in Fig. 12 are 2.58 for P1 and 2.88 for P2 (both relative to the corresponding average DB). These values are consistent with the intensity ratios reported from observations at $602 \mathrm{~nm}$ (e.g., Sobotka \& Hanslmeier 2005; Sobotka $\&$ Puschmann 2009). Individual UDs in the simulation can reach significantly higher intensities (see Fig. 13).

Maps of the vertical magnetic field and vertical velocity on surfaces of equal optical depth at $630 \mathrm{~nm}$ are given in Fig. 14. While the magnetic field distribution becomes more diffuse and homogeneous with height, the velocity remains rather intermittent. Strong downflows at the edges of the largest UDs at $\tau_{630}=1$ (left panels) can capture inclined magnetic field lines and drag them downwards, thereby creating a hairpin-like structure with a patch of reversed polarity, indicated by red color in the magnetic-field map. Quantitative information on the distributions is provided by the area histograms for the same opticaldepth levels shown in Fig. 15. The histograms at $\tau_{630}=0.01$ roughly represent the results that would be obtained by carrying out inversions of spectro-polarimetric observations in the neutral iron lines at $630.15 \mathrm{~nm}$ and $630.25 \mathrm{~nm}$. The histograms at $\tau_{630}=1$ are roughly similar to those at constant geometrical depth $z=0$ shown in the lower panel of Fig. 7, with a broad range of values in the UDs. At $\tau_{630}=0.1$, field strengths below $1000 \mathrm{G}$ are rarely found in UDs (particularly for those from P1) and at $\tau_{630}=0.01$, the UD distributions are further shifted to higher field strengths. For instance, $90 \%$ of the UDs of dataset P1 have field strengths between $1800 \mathrm{G}$ and $2200 \mathrm{G}$ at $\tau_{630}=0.01$. This effect is due to the elevation of the surfaces of constant optical depth in UDs (in comparison to the DB) and the cusp-like shape of the UD structure (cf. Schüssler \& Vögler 2006; Riethmüller et al. 2008a; Bharti et al. 2009). The larger, stronger UDs in the convective phase (dataset P2, green lines) reach higher into the umbral atmosphere (cf. Fig. 18) and show therefore still a stronger signature of the reduced field strength at $\tau_{630}=0.01$ than the UDs from P1. For both datasets, the distributions for UDs and DB approach each other as the optical depth decreases; this reflects the decreasing area fraction of the cusp-shaped UD structures with increasing height.

The corresponding area histograms of the vertical velocity given in Fig. 16 show rather weak motion in the DB: at all three $\tau$ levels, about $50 \%$ of DB shows almost no vertical flow while the rest of the area has velocities in the range $\pm 200 \mathrm{~m} \mathrm{~s}^{-1}$. The strong upflows creating the UDs have already been considerably braked when they reach the level $\tau_{630}=1$. Higher up at levels $\tau_{630}=0.1$ and $\tau_{630}=0.01$, high-velocity tails (up to $\sim \pm 1 \mathrm{~km} \mathrm{~s}^{-1}$ ) appear in the distributions. These are mostly due to the upward-directed, jet-like "valve flows" from the UD cusps (Choudhuri 1986; Schüssler \& Vögler 2006) and the corresponding return flows. The latter, however, may be affected by the presence of the closed upper boundary of the simulation box.

Figure 17 illustrates, by means of histograms, the properties of average vertical magnetic field and velocity at various optical depths, together with the average elevations of the opticaldepth levels for all UDs from the datasets P1 and P2. The magnetic field histograms show that, at all optical-depth levels, the expulsion of magnetic flux from the expanding upflow plumes underlying the UDs is more clearly reflected in the case of P2. However, in most cases the region of very low field strengths does not extend above the surface of $\tau_{630}=1$. The cusp shape of the vertical UD structure (see Fig. 2 of Schüssler \& Vögler 2006) leads to an increase of the field strength for the iso- $\tau$ surfaces above $\tau_{630}=1$. In addition, the UD area is defined by the intensity structure corresponding to a height near $\tau_{630}=1$; the cusp's cross section shrinks toward higher iso- $\tau$ surfaces, so that we progressively sample more of the strong-field region surrounding the plume. A similar situation is found for the upflows (second row), where the average velocities do not exceed a few hundred $\mathrm{m} \mathrm{s}^{-1}$ at $\tau_{630}=1$. At higher levels, the jet-like outflows along the cusps of the upflow plumes are seen. The distribution of upflows is shifted towards lower velocities for the UDs from P2, since bigger and flatter UDs show weaker outflows. This results from the fact that the height reached by the upflow plume is only weakly dependent on its size (since the stratification above optical depth unity is strongly subadiabatic), so that the cusp overlying a large UD is broader (has a larger aspect angle) and the whole structures thus is flatter. Therefore, the channelling of the upflowing matter into the top of the cusp is less efficient and the jet-like outflows are weaker. The downflows (third row) at the UD periphery (for $\tau_{630}=1$ ) and adjacent to the upflow jets (for the other levels) are similar in average magnitude for $\mathrm{P} 1$ and $\mathrm{P} 2$ while the distributions for $\mathrm{P} 2$ are somewhat broader.

The bottom row of Fig. 17 shows histograms of the relative height difference between the levels of constant optical depth in UDs (averaged over UD area) and in the mean DB, thus representing the elevation of the optical-depth levels in the UDs relative to the DB. While the mean elevations range between 60-90 km, the levels for the UDs from P2 are, on average, 10-20 km higher than the corresponding levels of UDs from $\mathrm{P} 1$. Thus, the P2 UDs tend to penetrate somewhat higher into the umbral photosphere than their counterparts from P1.

The elevation of the surfaces of constant optical depth above the upflow plumes tends to hide their magnetic and flow structure from spectroscopic observations (e.g., Degenhardt \& Lites 1993a,b). It is therefore not surprising that observational results for the magnetic and flow properties of UDs do not provide a unique picture. A reduced field strength in UDs has been repeatedly reported (e.g., Wiehr \& Degenhardt 1993; Socas-Navarro et al. 2004) and some authors also find indications for a decrease of field strength with depth (Riethmüller et al. 2008a; Bharti et al. 2009). Other studies (Sobotka \& Jurčák 2009; Watanabe et al. 2009b) do not show significantly lower field strengths in UDs. While upwellings in the deep layers of UDs were found (Socas-Navarro et al. 2004; Rimmele 2004; Riethmüller et al. 2008a; Bharti et al. 2007a, 2009), the jet-like upflows predicted by the simulations in the higher layers have not been observed 

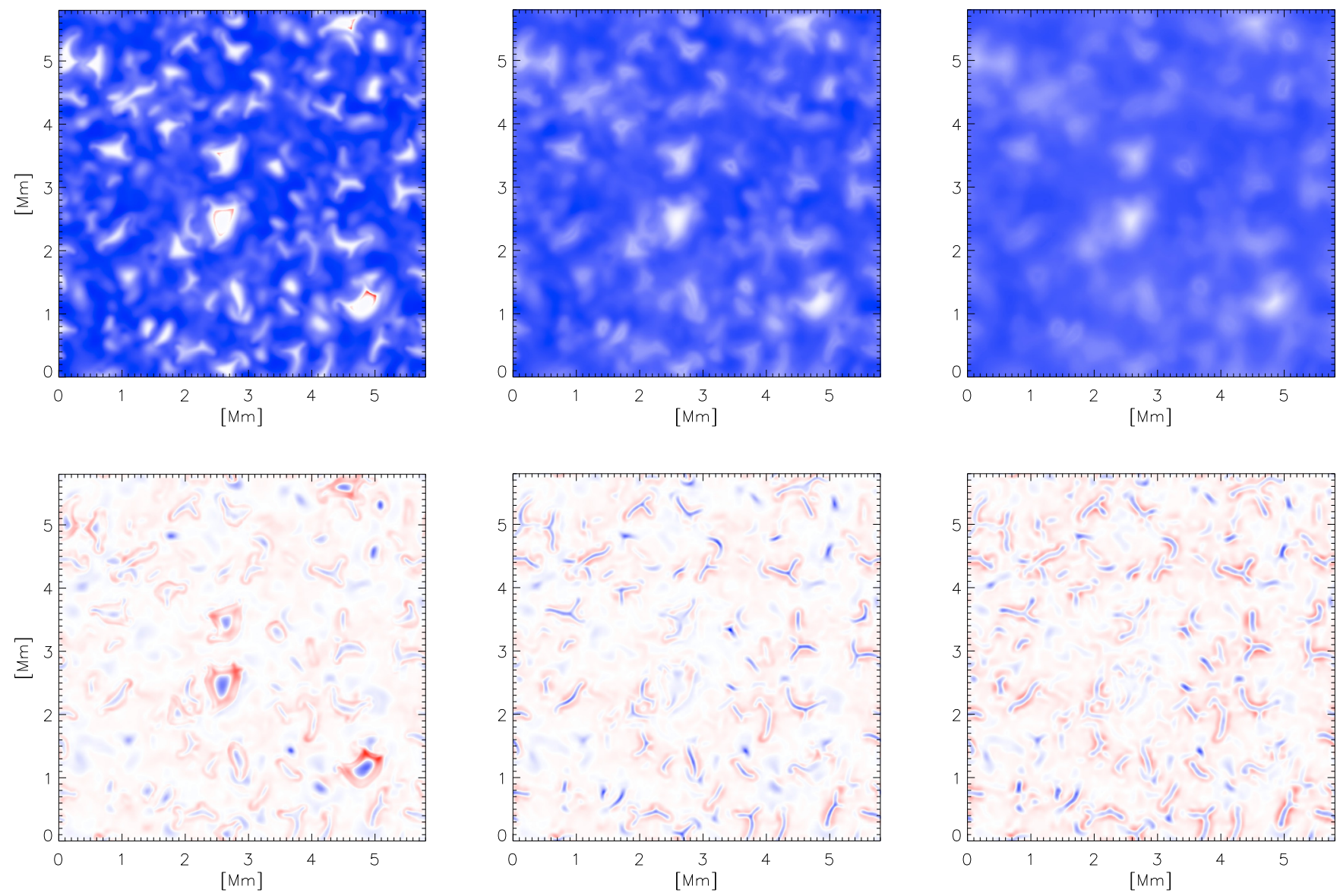

Fig. 14. Maps of vertical magnetic field strength (upper row) and vertical velocity (lower row) on surfaces of constant optical depth at $630 \mathrm{~nm}$ (from left to right: $\tau_{630}=1,0.1,0.01$, respectively). The color table for the magnetic field ranges from $3780 \mathrm{G}$ (blue) over zero (white) to $-190 \mathrm{G}$ (red). For the velocity the range is from $1.9 \mathrm{~km} \mathrm{~s}^{-1}$ (blue, upflow) over zero (white) to $-1.7 \mathrm{~km} \mathrm{~s}^{-1}$ (red, downflow).

so far. This may be due to the fact that spectroscopic studies mostly refer to large UDs, for which the jets are weaker or absent.

The relations between various mean quantities for the full set of UDs found by segmentation of continuum images at $630 \mathrm{~nm}$ are illustrated by scatter plots in Fig. 18. The positive correlation between UD area and brightness seen in Fig. 11 (for bolometric brightness) is confirmed also for $630 \mathrm{~nm}$ (top left panel). Although there is a large amount of scatter, UDs from P2 tend to be brighter for the same area, possibly reflecting higher upflow speeds (below optical depth unity) with a higher convective energy flux.

This interpretation is supported by the decrease of the magnetic field strength with increasing UD area at $\tau_{630}=0.1$ (top right panel) and with increasing intensity at $\tau_{630}=1$ (bottom left panel) for P2 as well as by the rise of the elevation of the level $\tau_{630}=1$ with increasing UD intensity (bottom right panel). The downflows at $\tau_{630}=1$ intensify with increasing brightness (middle right panel). The upflows $\tau_{630}=1$ are largely independent of brightness (middle left panel); since the level surface is higher for brighter UDs, this also indicates stronger underlying upflows.

\section{Conclusions}

The simulation of magneto-convective energy transport in a strong vertical magnetic field exhibits narrow upflow plumes, whose properties can be compared with observations of (central) umbral dots in sunspots. Using a multi-level image segmentation and tracking algorithm, we analyzed sets of simulated UDs from two phases of the simulation, the thermal relaxation phase (P1) and the quasi-stationary phase $(\mathrm{P} 2)$. This led to the following results:

1. Size: histograms of UD area indicate that there is no "typical" size of the simulated UDs. The average area is $0.08 \mathrm{Mm}^{2}$ in $\mathrm{P} 1$ and $0.14 \mathrm{Mm}^{2}$ in P2. Reported values from recent highresolution observations are somewhat lower. This is partly due to different size definitions, but could also indicate a lack of small, short-lived UDs in the simulation.

2. Brightness: averaged over their area, the bolometric brightness of the simulated UDs exceeds the surrounding dark background by a factor $1.6(\mathrm{P} 1)$ and $1.7(\mathrm{P} 2)$, respectively. The corresponding values for the continuum brightness at $630 \mathrm{~nm}$ are $2.6(\mathrm{P} 1)$ and $2.9(\mathrm{P} 2)$. For the peak intensities, the factors can reach markedly higher values between about 3 (bolometric) and $8(630 \mathrm{~nm})$. None of the simulated UDs exceeds the corresponding brightness values of the quiet Sun. Comparison with observations is complicated by the often unknown amount of straylight contamination.

3. Lifetime: average lifetimes for the simulated UDs are $28 \mathrm{~min}$ (P1) and $25 \mathrm{~min}$ (P2). Reported lifetimes from observations vary significantly, but recent high-resolution data typically indicate shorter lifetimes, although long-lived UDs are also found. It is possible that the simulations, owing to 

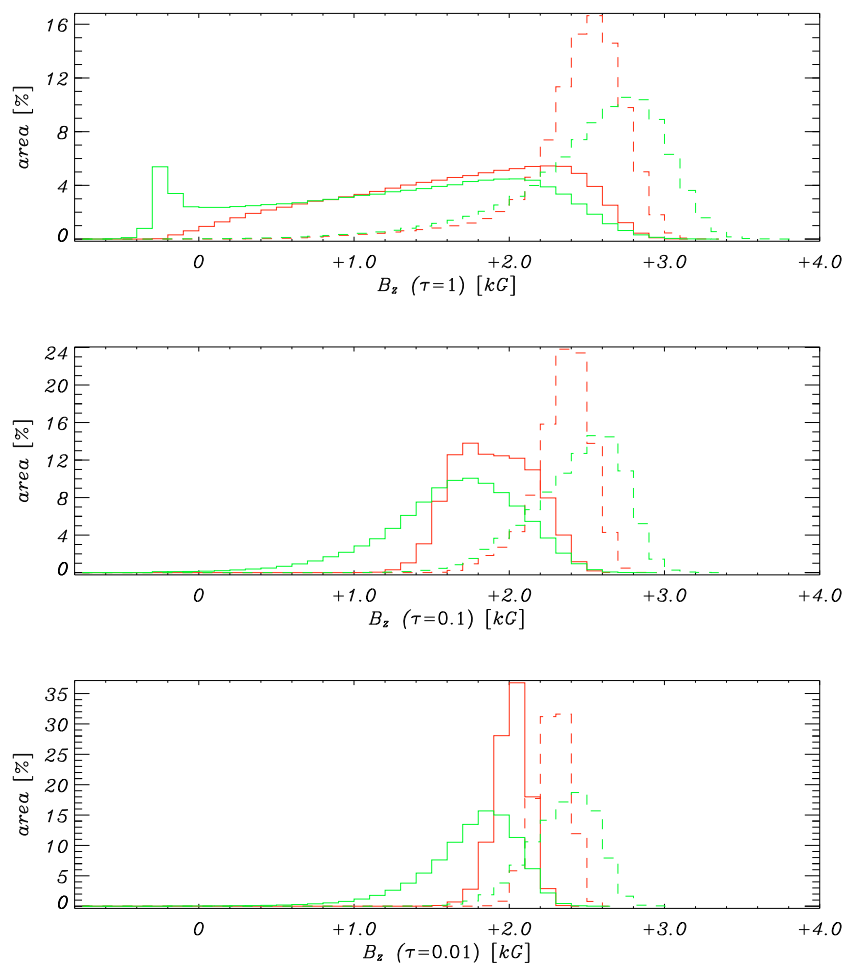

Fig. 15. Area histograms of the vertical magnetic field component on surfaces of constant optical depth at $630 \mathrm{~nm}$. Upper panel: $\tau_{630}=1$; middle panel: $\tau_{630}=0.1$; lower panel: $\tau_{630}=0.01$. Solid lines refer to UDs, dashed lines to the DB. The red and green colors indicate datasets $\mathrm{P} 1$ and $\mathrm{P} 2$, respectively.
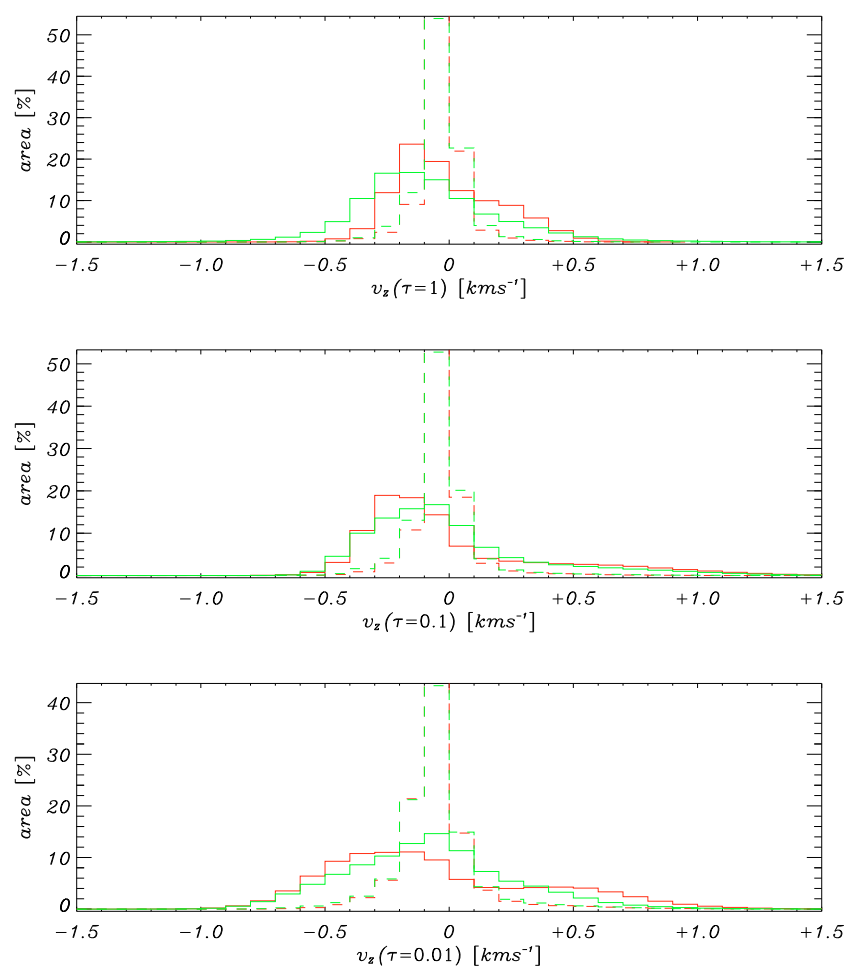

Fig. 16. Same as Fig. 15 but for the vertical component of the velocity. Positive velocity corresponds to upward motion, negative velocity to a downward flow.

limitations in spatial resolution, miss a population of small, short-lived UDs.
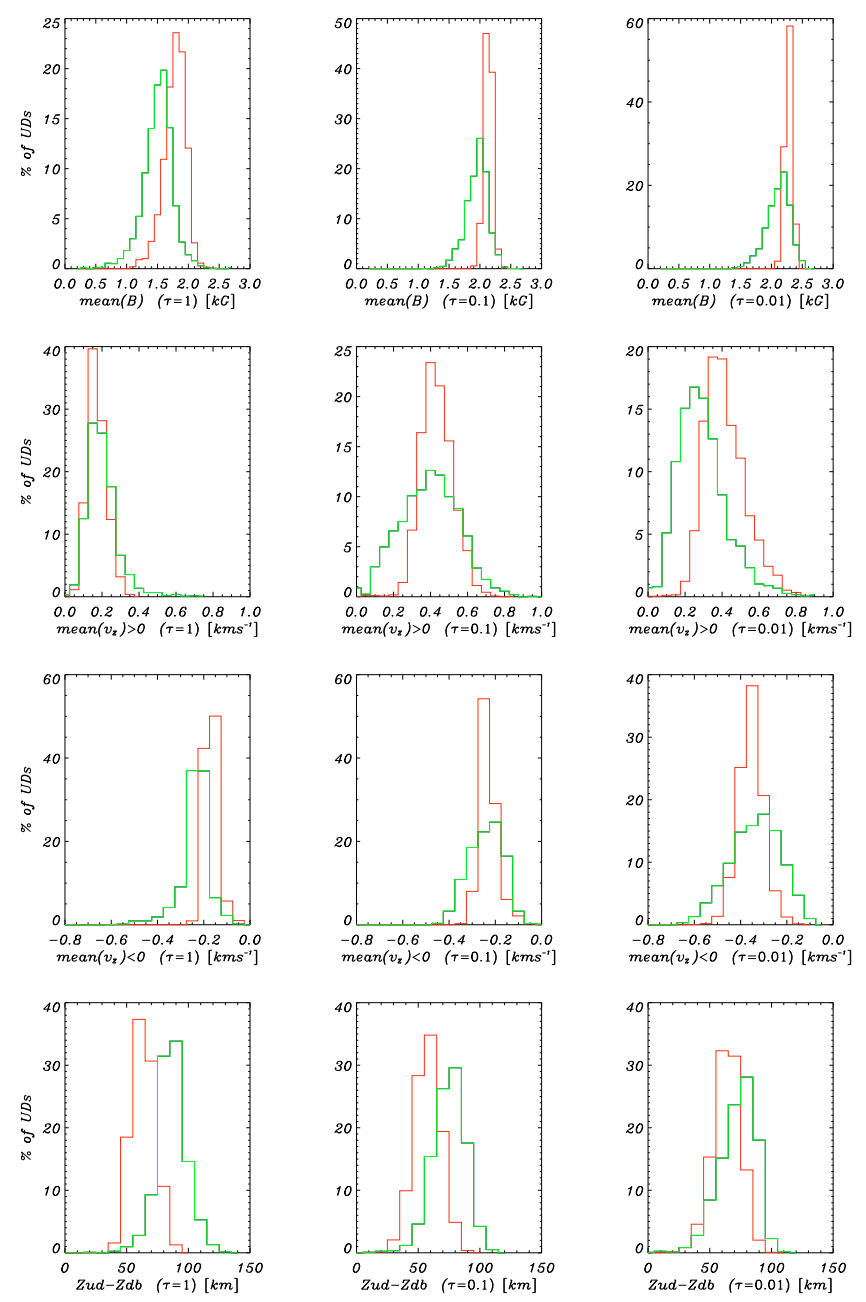

Fig. 17. Distributions of averages over UD area of vertical magnetic field (top row) and vertical velocity (separately for upflows, $v_{z}>0$, and downflows, $v_{z}<0$ in the second and third row, respectively), all on surfaces of constant optical depth at $630 \mathrm{~nm}$ (left column: $\tau_{630}=1$, middle column: $\tau_{630}=0.1$, right column: $\tau_{630}=0.01$ ). The bottom row gives the elevation of the optical-depth levels of the UDs with respect to the corresponding mean levels in the DB. Red and green lines refer to UDs from datasets $\mathrm{P} 1$ and $\mathrm{P} 2$, respectively.

4. Correlations: larger UDs tend to be brighter and live longer, although there is a significant amount of scatter. Similar trends have been reported from observations.

5. Magnetic field and flows: the drastic reduction of the magnetic field and the strong flows in the near-photospheric parts of the upflow plumes are largely hidden from spectropolarimetric observations. This is caused by the elevation of the surfaces of constant optical depth unity, which bulge upward over columns of hot rising plasma. Consistent with observational results, only a moderate field reduction and weak flow signatures are expected at optical-depth levels where relevant photospheric lines are formed.

In summary, the comparison of the properties of simulated and observed (central) UDs indicates that the simulations have caught the basic underlying mechanism for the formation of these bright structures. Differences in detail are not surprising, given the still unsufficient spatial resolution of both simulations and observations. Simulations of full sunspots, which have recently become available (Rempel et al. 2009a), show that 
L. Bharti et al.: Properties of simulated sunspot umbral dots
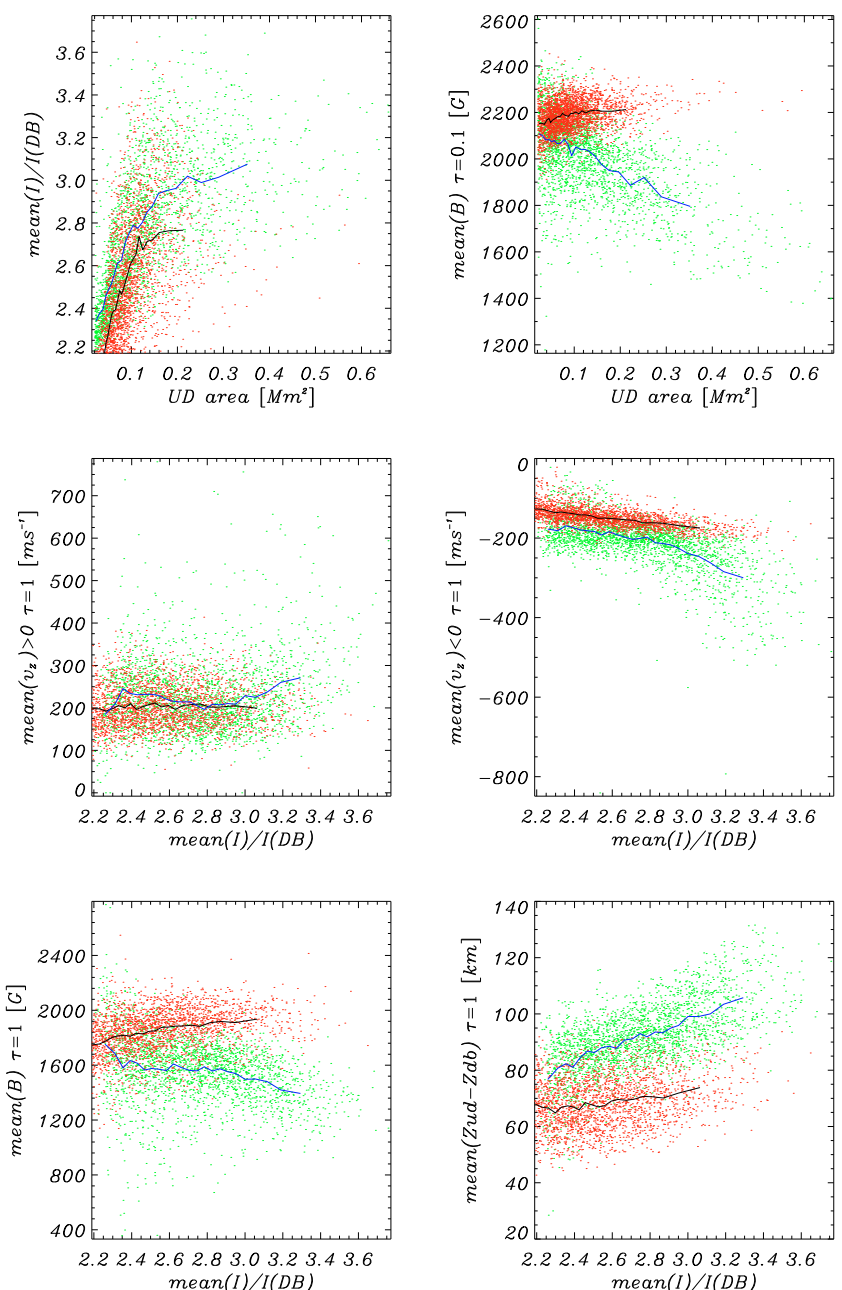

Fig. 18. Relationship between properties of UDs defined by segmentation of continuum images at $630 \mathrm{~nm}$. Mean quantities are averages over the area of the individual UDs. Red and green dots refer to datasets P1 and $\mathrm{P} 2$, respectively; solid black (P1) and blue (P2) lines connect binned values for 100 points each. Positive velocity corresponds to upward motion, negative velocity to a downward flow.

the similar magneto-convective processes are responsible for the formation of umbral dots and penumbral filaments, also clarifying the relationship between central and peripheral UDs.
More comprehensive parameter studies are needed to reveal the dependence of UD properties on background field strength, spatial resolution, and vertical extension of the computational box. Also, the calculation of synthetic Stokes profiles will permit a direct comparison with spectro-polarimetric observations and inversions.

\section{References}

Bharti, L., Jain, R., \& Jaaffrey, S. N. A. 2007a, ApJ, 665, L79

Bharti, L., Joshi, C., \& Jaaffrey, S. N. A. 2007b, ApJ, 669, L57

Bharti, L., Joshi, C., Jaaffrey, S. N. A., \& Jain, R. 2009, MNRAS, 393, 65

Bovelet, B., \& Wiehr, E. 2001, Sol. Phys., 201, 13

Bovelet, B., \& Wiehr, E. 2007, Sol. Phys., 243, 121

Bovelet, B., \& Wiehr, E. 2008, A\&A, 488, 1101

Choudhuri, A. R. 1986, ApJ, 302, 809

Degenhardt, D., \& Lites, B. W. 1993a, ApJ, 404, 383

Degenhardt, D., \& Lites, B. W. 1993b, ApJ, 416, 875

Ewell, Jr., M. W. 1992, Sol. Phys., 137, 215

Grossmann-Doerth, U., Schmidt, W., \& Schroeter, E. H. 1986, A\&A, 156, 347 Heinemann, T., Nordlund, Å., Scharmer, G. B., \& Spruit, H. C. 2007, ApJ, 669, 1390

Kitai, R., Watanabe, H., Nakamura, T., et al. 2007, Publ. Astron. Soc. Jpn., 59, 585

Kobel, P., Hirzberger, J., Solanki, S. K., Gandorfer, A., \& Zakharov, V. 2009, A\&A, 502, 303

Kusoffsky, U., \& Lundstedt, H. 1986, A\&A, 160, 51

Rempel, M., Schüssler, M., Cameron, R. H., \& Knölker, M. 2009a, Science, 325, 171

Rempel, M., Schüssler, M., \& Knölker, M. 2009b, ApJ, 691, 640

Riethmüller, T. L., Solanki, S. K., \& Lagg, A. 2008a, ApJ, 678, L157

Riethmüller, T. L., Solanki, S. K., Zakharov, V., \& Gandorfer, A. 2008b, A\&A, 492, 233

Rimmele, T. R. 2004, ApJ, 604, 906

Scharmer, G. B., Nordlund, Å., \& Heinemann, T. 2008, ApJ, 677, L149

Schüssler, M., \& Vögler, A. 2006, ApJ, 641, L73

Sobotka, M., \& Hanslmeier, A. 2005, A\&A, 442, 323

Sobotka, M., \& Jurčák, J. 2009, ApJ, 694, 1080

Sobotka, M., \& Puschmann, K. G. 2009, A\&A, 504, 575

Sobotka, M., Brandt, P. N., \& Simon, G. W. 1997, A\&A, 328, 689

Socas-Navarro, H., Pillet, V. M., Sobotka, M., \& Vázquez, M. 2004, ApJ, 614, 448

Tritschler, A., \& Schmidt, W. 2002, A\&A, 388, 1048

Vögler, A. 2003, Ph.D. Thesis, Univ. of Göttingen, Germany http: //webdoc. sub.gwdg.de/diss/2004/voegler

Vögler, A., Shelyag, S., Schüssler, M., et al. 2005, A\&A, 429, 335

Watanabe, H., Kitai, R., \& Ichimoto, K. 2009a, ApJ, 702, 1048

Watanabe, H., Kitai, R., Ichimoto, K., \& Katsukawa, Y. 2009b, Publ. Astron. Soc. Jpn., 61, 193

Wiehr, E., \& Degenhardt, D. 1993, A\&A, 278, 584

Wiehr, E., Bovelet, B., \& Hirzberger, J. 2004, A\&A, 422, L63 